\title{
To the issue of evaluating sonic boom overpressure and loudness
}

\author{
Igor G. Bashkirov ${ }^{1}$, Sergey L. Chernyshev ${ }^{1}$, Vladlen S. Gorbovskoy ${ }^{1}$, Andrey V. Kazhan ${ }^{1}$ *, \\ Vyacheslav G. Kazhan ${ }^{1}$, and Victor V. Kovalenko ${ }^{1}$ \\ ${ }^{1}$ Central Aerohydrodynamic Institute (TsAGI), 140180 Zhukovsky, Russia
}

\begin{abstract}
At present, in the world there is a growing interest in the development of a new generation of supersonic passenger aircraft. One of the main problems of creating such aircraft is to ensure both an acceptable sonic boom level and high aerodynamic characteristics in the supersonic cruising mode. This requires the development of reliable methods for obtaining the near field under the plane with taking into account the influence of the boundary layer, calculation of overpressure signature on the ground and evaluation of sonic boom loudness. In this work four variants of the equivalent body of revolution of minimum sonic boom with different nose sharpening were investigated for an aircraft weighing 19 tons in supersonic cruising flight at Mach number of 1.7 and altitude of $15.5 \mathrm{~km}$ using the software package for solving the Reynolds-averaged NavierStokes equations (RANS) ANSYS CFX. A macro for calculating the overpressure signature on the ground for the distribution of disturbances in the near field under the aircraft and a program for evaluating the sonic boom loudness in various metrics were developed. Computational mesh verification of the results was carried out, the obtained overpressure signatures were compared with theoretical data and calculation results from the software package for the integration of complete system of Euler equations by finite-difference method X-CODE. The effect of the sharpening of the nose part on aerodynamic drag and sound boom characteristics was shown. The work was done in the interests of the international project RUMBLE (RegUlation and norM for low sonic Boom LEvels).
\end{abstract}

\section{Introduction}

One of the challenges of modern aviation science is a creation of the new generation civil supersonic transport (SST), which can significantly reduce the flight time and increase the one-day travel range up to $7000-8000 \mathrm{~km}$. Research in this direction is carried out in the USA, Japan, the EU and Russia. The experience in operating supersonic passenger aircraft of the first generation (Tupolev Tu-144 and Concorde) has shown that with growing problems of safety, efficiency and environmental friendliness the long-range supersonic passenger aircraft require substantially higher operating costs compared to subsonic aircraft.

\footnotetext{
* Corresponding author: andrey.kazhan@tsagi.ru
} 
When developing the second-generation supersonic passenger aircraft, along with the search efforts for new technical solutions and research on aerodynamics, power plant and structural materials providing a high level of technical and economic parameters, great attention is paid to minimizing the environmental impact - decreasing to acceptable levels of sonic boom during supersonic cruise flight and the airport noise at take-off and landing as well as reducing the destructive effect on the Earth ozone layer. It is worth noting that at present, in a number of countries there is a ban on flying over their territories at supersonic speeds due to the negative impact of sonic boom.

Research over the past 30 years has shown that for business jets it is possible to provide an acceptable level of sonic boom for flights over inhabited land by performing a number of measures concerning aerodynamic layout and power plant arrangement. This led to the emergence of several projects of supersonic business aircraft, such as Aerion, QSST, Gulfstream QSJ, Spike, Boom (USA), as well as Russian SSBJ projects of Sukhoi Civil Aircraft Company, Tu-444 of Tupolev JSC etc. At the same time, under the auspices of ICAO, work is underway to create standards for an acceptable level of sonic boom and noise near the airport for perspective supersonic passenger aircraft (NASA and Lockheed Martin QueSST projects [1], JAXA D-SEND [2], the European Union and Russia RUMBLE [3]). These projects are aimed at determining the level of impact of sonic boom of various intensity on the environment, people, buildings and structures, including the use of low-boom technology demonstrators, and at developing computational methods to predict the propagation of sonic boom in real atmosphere [4].

This work is devoted to the development of approaches used at TsAGI to calculate the overpressure signature and sound boom loudness. A method to determine the overpressure in the "near field" around the aircraft using modern methods of computational gas dynamics is presented, its verification and validation is performed based on the materials of AIAA Sonic Boom Prediction Workshop [4]. The example of equivalent bodies of revolution of minimum sonic boom shows the effect of sharpening of the body nose on the aerodynamic drag characteristics and sonic boom intensity is shown.

\section{Method for calculating the overpressure signature}

Due to the lack of real data on the propagation of a low-intensity sonic boom occurring during flight at supersonic speeds in real atmosphere, the calculation methods have been widely used to evaluate the overpressure distribution on the ground and the level of sonic boom loudness. The traditional calculation procedure consists of several stages [5]:

- Using the means of computational gas dynamics, the flow around an aircraft in the so-called "near field" is calculated. As a rule, the computational domain contains a cylinder with a diameter of D 5 aircraft lengths, which, according to the sonic boom theory, is sufficient to set an asymptotic, that is, independent of the angular coordinate, solution. By solving the Euler equations in an inviscid formulation or the Reynolds-averaged Navier-Stokes equations for calculating with regard to viscosity, the pressure distribution on the outer surface of the computational domain is determined.

- From the obtained pressure distribution in any azimuth plane using nonlinear acoustics approximations, the distribution of overpressure signature is calculated with regard to attenuation in the atmosphere. In this case, it is possible to take into account the real atmospheric parameters, such as humidity, temperature, wind, etc.

- The loudness of the signal that came to the ground is calculated taking into account the acoustical reflection from the ground by means of Fourier transform with further correction of the spectrum according to the chosen metrics.

The task of calculating the flow in the "near field" has its own peculiarities. For a detailed description of the local areas of pressure change and minimization of the effect of "numerical 
viscosity" on the overpressure signature at a sufficiently large distance from the aircraft $(\mathrm{H} / \mathrm{L} \sim 5)$, it is necessary to create detailed computational meshes, oriented along the Mach cone corresponding to the flow velocity. This, in turn, requires the creation of unique computational meshes for each flight mode with variations in speed and angle of attack.

An alternative approach to determining the overpressure signature in the "near field" is to use the Whitham function [6], which can be calculated for a specific azimuth plane from the distribution of the area of the corresponding equivalent body of revolution:

$$
\bar{F}(\bar{\eta})=\int_{0}^{\bar{\eta}} \frac{\bar{s}_{e q}^{\prime \prime}(\bar{x})}{\sqrt{\bar{\eta}-\bar{x}}} d \bar{x}
$$

where $\bar{\eta}$ is the distance along the body axis related to the characteristic length $L, \bar{x}=x / L$ is the relative coordinate along the body axis, $S_{e q}(x)$ is the area of an equivalent body of revolution, which creates the same disturbances in the near field as the aircraft in question.

When calculating the area distribution of the equivalent body of revolution, both geometric and lifting characteristics of the aircraft are taken into account (Fig. 1a):

$$
S_{e q}(x)=S_{A}(x)+S_{Y}(x)=S_{A}(x)+\frac{\beta}{2 q} \int_{0}^{\eta} Y(x) d x
$$

where $S_{A}$ is a square of YZ projection of the reverse Mach plane section of an airplane, $\beta=$ $\sqrt{\mathrm{M}_{\infty}^{2}-1}, \mathrm{M}_{\infty}$ is the free-stream Mach number, $q$ is the velocity head, $Y(x)$ is sectional lift.

The exact calculation of area components of the equivalent body of revolution for the geometry of an aircraft of complex shape causes certain difficulties. TsAGI has developed and uses an alternative technique [7-9] to determine $S_{e q}(x)$ when disturbances from the body streamlined by supersonic flow are quite small. Fig. $1 \mathrm{~b}$ shows the control volume of the supersonic flow around the wing-fuselage layout and $S_{1}$ plane separates the disturbed flow from the undisturbed one. The $\mathrm{S}_{2}$ plane is parallel to the $x$ axis and is at some distance under the body. The AB segment is on the line of intersection of the surface $S_{1}$ and the plane $S_{2}$ and defines the characteristic surfaces $\mathrm{S}_{2}$ and $\mathrm{S}_{4}$, which are the envelopes of reversed Mach cones. It is shown in [7] that for the direction straight downwards under the plane for the distribution of the area of an equivalent body of revolution the following relation can be obtained:

$$
\frac{d S_{e q}(x)}{d x}=-\frac{1}{V_{\infty}} \int_{A}^{B}(\beta u+v) d z=-\frac{2 \beta}{V_{\infty}} \int_{A}^{B} u d z=-\frac{2}{V_{\infty}} \int_{A}^{B} v d z
$$

where $u$ and $v$ are components of the perturbed velocity along axes $x$ and $y$, respectively.
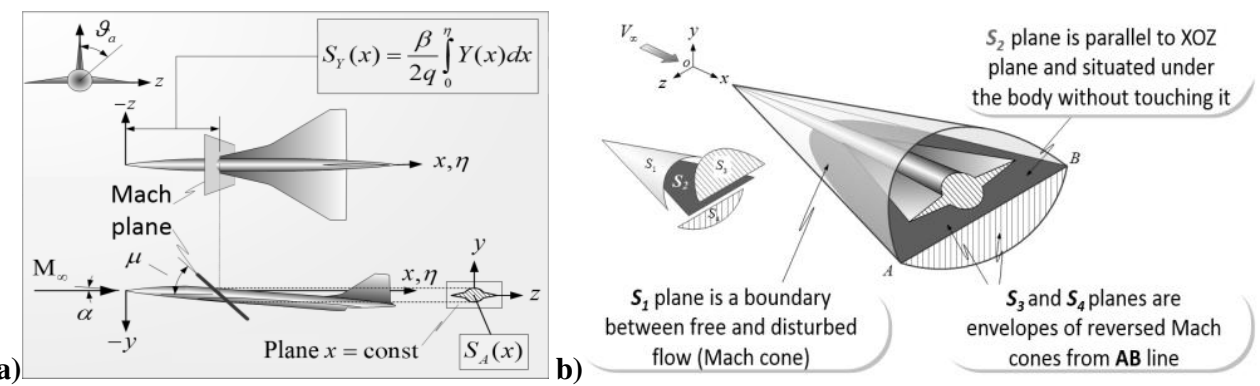

Fig. 1. a) Area components of the equivalent body; b)Control volume around a body

Thus, the distribution of the area of the equivalent body of revolution can be determined if the perturbed velocity components are known at any, even a small distance from the streamlined body. In this paper, this approach is applied to the calculation of flow around supersonic aircraft using a commercial software package for solving RANS equations 
ANSYS CFX. A macro has been developed for this software, allowing for distribution of the equivalent body of revolution area derivative using the results of flow simulation and calculation of Whitham function. It is worth noting that the above approach can be used for calculation of sonic boom characteristics simultaneously with the assessment of aircraft aerodynamic characteristics for any flight mode without rebuilding the computational mesh.

To calculate the propagation of the overpressure signature in the atmosphere, a system of equations obtained in [10] using the algorithms formulated in [11] is used:

$$
\begin{array}{rlrl}
\Delta P & =k_{1} \bar{F}(\bar{\eta}), & t=k_{2}[\bar{\eta}-k \bar{F}(\bar{\eta})], \\
\left(\overline{F_{2}}-\overline{F_{1}}\right) k=\bar{\eta}_{2}-\bar{\eta}_{1}, & \left(\overline{F_{2}}+\overline{F_{1}}\right)\left(\bar{\eta}_{2}-\bar{\eta}_{1}\right)=2 \int_{\bar{\eta}_{1}}^{\bar{\eta}_{2}} \bar{F} d \bar{\eta}
\end{array}
$$

where $\Delta P$ is the overpressure and $t$ is the time in the overpressure signature, $\bar{F}(\bar{\eta})$ is the Witham function from equation (1), the coefficients $k, k_{1}$ and $k_{2}$ determine the attenuation of disturbances in an atmosphere.

To calculate the propagation of the overpressure signature, it is possible to use alternative computation codes, for example, ZEPHYRUS [12], which allows taking into account the absorption and dispersion of sonic waves in the atmosphere.

\section{Verification and validation of the calculation method}

\subsection{Objects of research}

As objects of research four variants of bodies of revolution of the minimum sonic boom with parameters corresponding to flight of a light supersonic executive airplane with a length $\mathrm{L}=23 \mathrm{~m}$ and weight at the beginning of cruising flight $\mathrm{G}_{\text {cruise }}=19 \mathrm{t}$ are considered in this paper. The cruising flight is performed at an altitude $\mathrm{H}=15.5 \mathrm{~km}$ at a speed corresponding to Mach number $\mathrm{M}=1.7$. The geometry of the bodies of the minimum sonic boom is obtained using the method described in [13] and differs in various nose sharpening (Fig. 2).
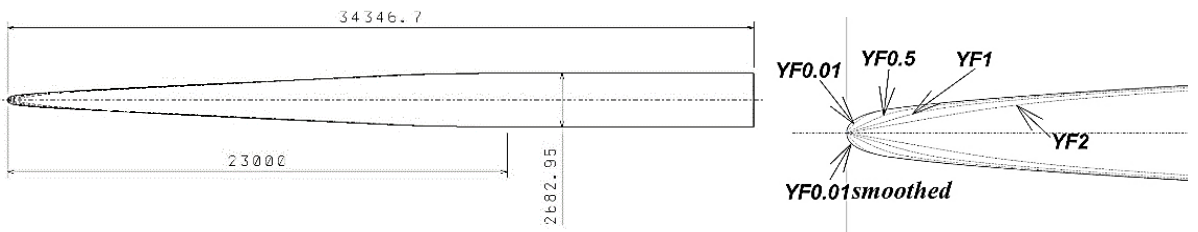

Fig. 2. Geometry of the studied variants of bodies of revolution of minimum sonic boom

\subsection{Calculation method verification}

Verification of the described technique with respect to computational mesh refining parameters was carried out on the body with the smallest sharpening of the nose, since the flow about this geometry may cause the formation of a local subsonic zone behind the bow shock, which can significantly affect the flow parameters downstream. The influence of computational mesh density both on the sonic boom characteristics, namely, on the overpressure signature on the ground, and on the value of aerodynamic drag was investigated.

Unstructured tetrahedral computational meshes with prism-layers near the body surface were used for the calculation to describe the flow in the boundary layer. The computational mesh refining domain was a truncated cylinder around the examined body. With a characteristic body length $\mathrm{L}=23 \mathrm{~m}$ and a diameter of its end part $\mathrm{D}=2.682 \mathrm{~m}$, the following 
values of the maximum cell size inside the refining domain are considered: 400, 200, 150, $100,75,65$, and $55 \mathrm{~mm}$. The integration of perturbed velocities to determine the derivative of the equivalent body of revolution was carried out on planes located under the body axis at distances corresponding to 1,3 and 5 body diameters.

In Figs. 3 a) and b) the verification results of the calculation by the number of computational mesh nodes are shown. Fig. 3 a) shows the aerodynamic drag coefficient, and Fig. $3 \mathrm{~b}$ ) shows the maximum pressure in signatures on the ground, calculated by the disturbances at different distances from the body in comparison with theoretical data.
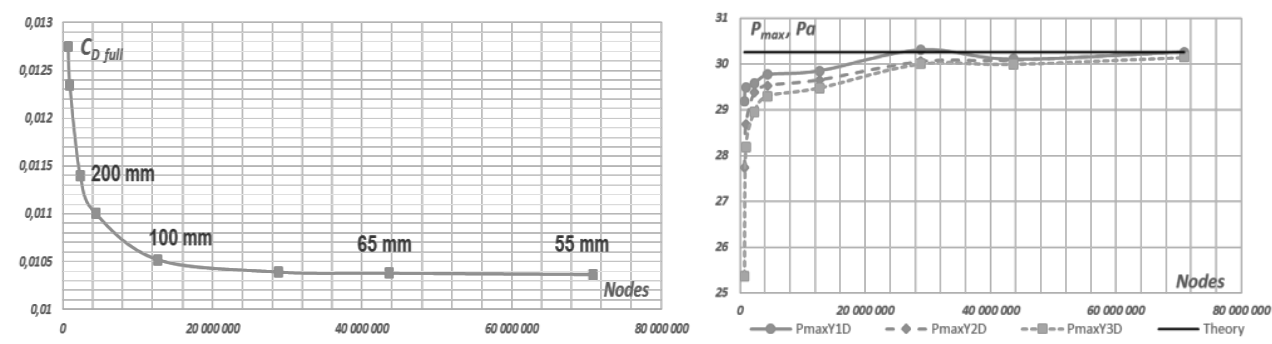

Fig. 3. a) Aerodynamic drag coefficient vs mesh density; b) Maximum pressure drop in the signature vs mesh density

As a result of the studies, it was found that for a given size of the investigated body, the solution weakly depends on further mesh refinement at maximum size of the element less than $75 \mathrm{~mm}$ in refining domain. Further calculations were performed on computational meshes with a refining parameter of $55 \mathrm{~mm}$. The values of the velocity perturbations for calculating the derivative of the equivalent body of revolution were taken on the plane under the body at a distance corresponding to $\mathrm{H} / \mathrm{L}=0.1$.

\subsection{Calculation method validation}

Validation of the method for calculating the overpressure signature at sonic boom was carried out using the materials of the AIAA Sonic Boom Prediction Workshop (SBPW) [4]. Both the geometry of the AXIE body of revolution and the geometry of aerodynamic layout of NASA C25P low-sonic boom aircraft with simulation of the power plant operation were considered. Comparison of the results was based on the distribution of pressure at the "near field" boundary at $\mathrm{H} / \mathrm{L}=5$ and the dependence of the overpressure on time on the ground.

The calculation results are presented in Fig. 4 for the AXIE body and in Fig. 5 for NASA $\mathrm{C} 25 \mathrm{P}$. It can be seen that the results obtained using TsAGI technique are in good agreement with SBPW materials, especially in the front part of signatures, which indicates the correctness of the method used. The differences in the rear part of signatures for the aircraft configuration with operating power plant are apparently related to the interference of trailingedge shock waves and the jet stream and their propagation to the "near field" boundary.
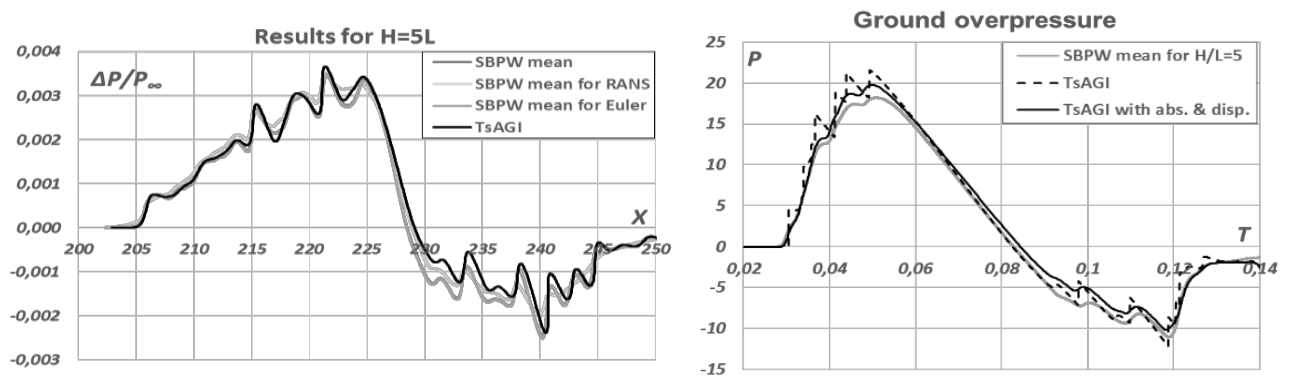

Fig. 4. Comparison of results for AXIE body of revolution 

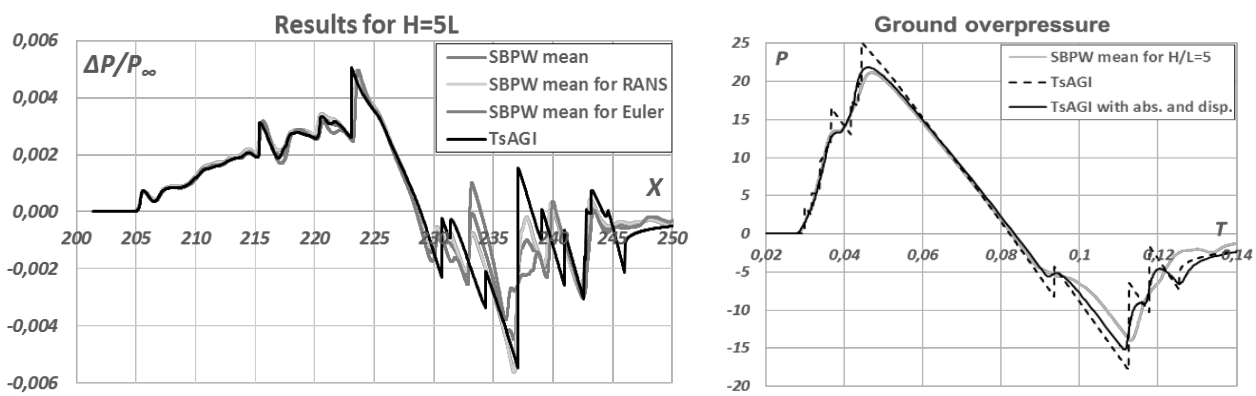

Fig. 5. Comparison of results for NASA C25P aircraft configuration

As noted earlier, one of the advantages of this approach is the ability to carry out calculations on the same computational mesh for different flight modes with a simultaneous assessment of aircraft aerodynamic characteristics. Table 1 presents a comparison of the calculated aerodynamic forces and moments coefficients with SBPW data.

Table 1. Comparison of aerodynamic characteristics.

\begin{tabular}{||l|c|c||}
\hline \hline Value on $\boldsymbol{\alpha}=\mathbf{0}^{\circ}\left(\boldsymbol{\alpha}_{\text {real }}=\mathbf{3 , 3 7 5}\right)$ & SBPW (M. Park, NASA) & TSAGI \\
\hline$C_{D}$ (without power plant) & 0,01442 & 0,01446 \\
\hline$C_{D}$ (with power plant) & 0,03292 & 0,03296 \\
\hline$C_{L}$ & 0,05356 & 0,0544 \\
\hline$m_{z}$ (relative nose) & $-0,03921$ & $-0,04065$ \\
\hline
\end{tabular}

\section{Calculation of sonic boom loudness}

To quantify the level of sonic boom impact on a person, a characteristic of sonic boom loudness is used. The auditory perception of a person is a non-linear and frequency-dependent characteristic. Therefore, to calculate the loudness level, the frequency spectrum of the acoustic signal is multiplied by some weight function, taking into account the level of perception by the ear of various frequencies. Currently, studies are being carried out on the applicability of different loudness metrics (A, B, C, D, PL, ISBAP) to adequately describe the level of sonic boom impact on a person [3]. In general, the calculation of the acoustic signal loudness in A, B, C metrics is based on the estimation of the excitation energy [14]. Using the Fourier transform to decompose the signal $P(t)$ into a spectrum in frequencies $P(\omega)$ and multiplying the obtained spectrum by the weight function $\Omega_{\mathrm{x}}(\omega)$ we can calculate the loudness in the corresponding metric:

$$
L_{x}=10 \lg \left[\frac{1}{t_{0}}\right] \int_{t_{1}}^{t_{2}} \frac{P_{x}^{2}(t)}{P_{r e f}^{2}} d t=10 \lg \left[\frac{1}{\pi}\right] \int_{\omega_{1}}^{\omega_{2}}\left|\frac{P(\omega)}{P_{r e f}} \Omega_{x}(\omega)\right|^{2} d \omega=
$$

where $L_{x}$ - sound pressure level; $x$ - metric index; $t_{0}$ - characteristic time equal for most metrics to $1 \mathrm{sec} ; P_{x}(t)-x$-weighted pressure distribution function; $P_{r e f}=2 \times 10^{-5} \mathrm{H} / \mathrm{m}^{2}-$ reference pressure level corresponding to the hearing sensitivity threshold.

However, a characteristic feature of the pressure wave during sonic boom is a pressure rise in front and rear parts of signature, which leads to the necessity of introducing corrections for the signal impulse $[15,16]$. Such corrections are implemented in PL (Perceived level) metric and consist in setting the characteristic time $t_{0}=0.07 \mathrm{sec}$ (experimentally determined critical time for which the human ear is able to fully perceive the incoming acoustic disturbance) and taking into account the energy correction. This correction arises from the assumption that most of the acoustic energy during sonic boom is contained in bow and 
breakdown shocks, which act after a period of time longer than the characteristic time $t_{0}$ and are perceived by the ear as two independent events. Since the acoustic energy in equation (5) is determined over the entire wave, assuming that the bow and the breakdown shocks are approximately the same, the spectral energy in PL metric is calculated with a coefficient of $1 / 2$. The loudness levels in $1 / 3$-octave frequency bands are determined by the formula:

$$
L_{x}=10 \lg \left[\frac{1}{\pi}\right]\left[\frac{1}{0.07}\right] \int_{\omega_{1}}^{\omega_{2}}\left|\frac{1}{2} \frac{P(\omega)}{P_{\text {ref }}}\right|^{2} d \omega=10 \lg \left[\frac{1}{\pi}\right]\left[\frac{1}{0.07}\right] \int_{\omega_{1}}^{\omega_{2}}\left|\frac{P(\omega)}{P_{\text {ref }}}\right|^{2} d \omega-3
$$

and for calculating the loudness, an algorithm MARK VII is used to convert equivalent loudness levels into Sones [17, 18].

Algorithms for calculating the sonic boom loudness in various metrics are implemented in TsAGI in a special program code validated within the RUMBLE project [3]. With this code loudness of any signal $P(t)$ can be calculated and it was used in further investigations.

It is worth noting that the sonic boom loudness depends significantly on the time of pressure rise in the shock wave, which in turn depends on the state of the atmosphere (turbulence, humidity, temperature, wind, etc.). Thus, the loudness change in PL metric when the pressure rise time in the bow shock of $30 \mathrm{~Pa} \mathrm{~N}$-wave increases from $\tau_{1}=1 \mathrm{~ms}$ to $\tau_{1}=5 \mathrm{~ms}$ is $\sim-7 \mathrm{PLdB}$, which is equivalent to $\sim 5$ times reduction of the perceived noise level. In this case, due to the small amount of experimental data for overpressure signature at sonic boom of low intensity, the task of determining the time of pressure rise in the overpressure signature in real atmosphere is extremely relevant and requires further research.

\section{Calculation of sonic boom and aerodynamic drag characteristics for bodies of revolution}

The above methods for calculating the loudness and overpressure signature are used to evaluate the characteristics of sonic boom when flowing around bodies of revolution of minimum sonic boom described in Section 3.1.

The sharpening of the nose has a noticeable effect on the shape of the nose compression wave and the flow parameters downstream, which affects both the sonic boom characteristics and the aerodynamic drag (Table 2). It should be noted that an increase in the degree of sharpening could reduce the body aerodynamic drag (up to $10 \%$ ) with a relatively small increase in the sonic boom loudness (less than $1 \mathrm{~dB}$ ).

Table 2. Comparison of bodies of revolution characteristics.

\begin{tabular}{||l|c|c|c|c|c||}
\hline \hline & YF0.01 & YF0.01smoothed & YF0.5 & YF1 & YF2 \\
\hline $\boldsymbol{C}_{\mathbf{D}}$ & 0,006433 & 0,006327 & 0,006201 & 0,006015 & 0,005833 \\
\hline $\boldsymbol{A} \boldsymbol{P}_{\boldsymbol{1}}, \boldsymbol{P a}$ & 18,77 & 19,08 & 20,05 & 20,97 & 21,95 \\
\hline $\boldsymbol{P}_{\max }, \boldsymbol{P a}$ & 30,27 & 30,26 & 29,25 & 29,59 & 30,23 \\
\hline $\boldsymbol{L}_{\boldsymbol{A}}, \boldsymbol{d} \boldsymbol{B} \boldsymbol{A}$ & 83,35 & 83,40 & 83,52 & 83,67 & 84,06 \\
\hline $\boldsymbol{L}_{\boldsymbol{P L} \boldsymbol{}}, \boldsymbol{P L} \boldsymbol{d} \boldsymbol{B}$ & 96,78 & 96,87 & 96,93 & 97,12 & 97,57 \\
\hline \hline
\end{tabular}

\section{Conclusion}

Creation of the new generation of civil supersonic aircraft requires adopting of international standards and developing technical solutions to ensure the reduction to acceptable levels of sonic boom and airport noise, aerodynamic and weight efficiency, etc. In the absence of real flight data for an aircraft with a low level of disturbances at the preliminary design, methods of computational fluid dynamics are widely used to evaluate the overpressure and sonic boom 
loudness on the ground. Nonlinear acoustics approaches are used to calculate the shock waves propagation in the atmosphere.

The method for calculating the disturbances in the "near field" around the aircraft developed at TsAGI is based on the calculation of distribution of perturbed velocity components at a small distance from the streamlined body to build an equivalent body of revolution. The method has been adapted to commercial ANSYS CFX software, its verification and validation has been performed. It was shown that the results are in good agreement with the data of AIAA Sonic Boom Prediction Workshop. One of the main advantages of this approach is the possibility to use one computational mesh with a moderate number of nodes for all flight modes and simultaneously determine both the sonic boom and aerodynamic characteristics. In addition, with this approach, the effects of "numerical viscosity" do not significantly affect the calculation results.

A program code has been developed and validated for calculating the loudness of sonic boom in various metrics. A number of loudness calculations had shown that the time of pressure rise in overpressure signature has a decisive influence on the level of sonic boom loudness. The problem of determining the time of pressure rise in the overpressure signature in real atmosphere is highly relevant and requires further research.

Investigation of bodies of revolution of the minimum sonic boom shows that the sharpening of the nose part has a weak effect on the sonic boom level, but leads to a noticeable decrease in aerodynamic drag. For the next generation supersonic passenger aircraft a compromise must be found between the level of sonic boom and aerodynamic characteristics, taking into account the strength, control system, power plant, etc.

\section{References}

1. http://www.nasa.gov/lowboom

2. http://www.aero.jaxa.jp/eng/research/frontier/sst/d-send.html

3. https://rumble-project.eu

4. https://lbpw.larc.nasa.gov

5. M. Park, M. Nemec, 35th AIAA Applied Aerodynamics Conference, p. 3256 (2017)

6. G.B. Whitham, Proc. Roy. Soc., ser. A, Vol. 201, N 1064 (1950)

7. Yu.L. Zhilin, V.V. Kovalenko, TsAGI Science Notes, Vol. XXIX, N 3-4, p. 111 (1998)

8. S.L Chernyshev, Sonic boom (Nauka, Moscow, 2011)

9. L.G. Ivanteeva, V.V. Kovalenko, E.V. Pavlyokov, L.L. Teperin, R.G. Racl, J. Acoust. Soc. Am., Vol. 111, No. 1, Pt. 2 (2002)

10. Yu.L. Zhilin, TsAGI Science Notes, Vol. II, N 3 (1971)

11. Yu.L. Zhilin, S.L. Chernyshev, TsAGI Works, Vol. 2110, p. 8 (1981)

12. L.D. Robinson, Ph.D. dissertation (University of Texas, 1991)

13. R.J. Mack, NASA/TM-2003-212653 (2003)

14. A.G. Munin, V.E. Kvitka, Avaition acoustics (Mashinostroenie, Moscow, 1973)

15. D. Johnson, D. Robinson, Acta Acustica, Vol. 21, No. 6, pp. 307-318 (1969)

16. K.P. Shepherd, B.M. Sullivan, NASA/TP-3134 (1991)

17. S. Stevens, The J. of the Ac. Soc. of Am., Vol. 51, No. 2B, pp. 575-601 (1972)

18. G. Jackson, H. Leventhall, Applied Acoustics, Vol. 6, No. 1, pp. 23-34 (1973) 